\title{
Pesquisa Nacional de Saúde, análises laboratoriais e monitoramento de metas de redução de Doenças Crônicas
}

A Pesquisa Nacional de Saúde (PNS) destaca-se por ser o mais abrangente inquérito populacional em saúde realizado no País e conta com duas edições 2013 e 2019. A pesquisa foi conduzida pelo Instituto Brasileiro de Geografia e Estatística (IBGE) em parceria com o Ministério da Saúde e em colaboração com instituições de ensino e pesquisa. A PNS inclui temas sobre determinantes e condicionantes em saúde, acesso e utilização de serviços, doenças crônicas, violências, fatores de risco, ciclos de vida, medidas antropométricas, além de ter inovado, em 2013, incluindo a coleta da medida da pressão arterial e material biológico (sangue e urina) em uma subamostra de cerca de 9.000 participantes.

Este suplemento da Revista Ciência e Saúde Coletiva contém artigos baseados em resultados dos exames laboratoriais da PNS 2013, e contribui para avançar nos conhecimentos sobre a saúde da população brasileira. Dentre as análises, a comparação entre os dados autorreferidos e as medidas bioquímicas de Diabetes Mellitus, doença renal crônica (DRC) e Colesterol, apontam que as medidas autorreferidas tendem a subestimar a prevalência das doenças, e conclui-se pela necessidade de incorporar o componente laboratorial na rotina dos inquéritos em saúde. Dados recentemente analisados e publicados amostram que a DRC é subestimada no País e as estimativas laboratoriais apresentam prevalências 4 vezes mais elevadas entre adultos ${ }^{1}$.

Neste suplemento, análises de diferentes calculadoras para estimar o Risco Cardiovascular (RCV) da população brasileira identificaram grande variação na proporção de indivíduos classificados como alto RCV, implicando em dificuldades na escolha da população alvo para priorização das políticas públicas. Os resultados indicam a necessidade de se avançar na definição dos valores de referência dos exames laboratoriais brasileiros, a partir de estudos nacionais.

Os resultados dos exames laboratoriais da PNS aqui apresentados, bem como das análises de inquéritos populacionais, possibilitam monitorar as metas dos Planos de Ação de Enfrentamento das DCNT. Desde 2015, com o aumento das desigualdades e as políticas de austeridade implantadas, ocorreram pioras nos indicadores de fatores de risco (FR) de DCNT (tabaco, consumo de frutas e hortaliças, atividade física, obesidade). Estes dados reforçam a importância de avançar em ações e políticas de promoção à saúde e medidas regulatórias para enfrentamento das DCNT.

A Revista C\&C tem destacado a importância da PNS no contexto da saúde pública brasileira, provendo informações oportunas, de qualidade, que podem orientar o planejamento em saúde, futuras direções, avanços e desafios. Muitas outras análises serão possíveis com esta importante base de dados, visando buscar respostas para a saúde coletiva. Destacamos ainda que na PNS 2019 não foi coletado o componente laboratorial, portanto a base da PNS 2013 permanece ímpar, sendo um convite para novas análises, em especial visando explorar aspectos referentes aos determinantes sociais e às desigualdades em saúde. Destacamos ainda a necessidade de incorporar este componente em futuras pesquisas, bem como a defesa da sustentabilidade desta importante pesquisa para a Saúde Pública brasileira.

Deborah Carvalho Malta (http://orcid.org/0000-0002-8214-5734) ${ }^{1}$

Célia Landman Szwarcwald (http://orcid.org/0000-0002-7798-2095) ${ }^{2}$

Cimar Azeredo Pereira (https://orcid.org/0000-0001-6183-1607) ${ }^{3}$

${ }^{1}$ Departamento de Enfermagem Materno-Infantil e Saúde Pública, Escola de Enfermagem, Universidade Federal de Minas Gerais. Belo Horizonte MG Brasil.

${ }^{2}$ Instituto de Comunicação e Informação Científica e Tecnológica em Saúde, Fiocruz. Rio de Janeiro RJ Brasil.

${ }^{3}$ Instituto Brasileiro de Geografia e Estatística. Rio de Janeiro RJ Brasil.

\section{Referências}

1. Malta DC, Machado IE, Pereira CA, Figueiredo AW, Aguiar LK, Almeida WS, Souza MFM, Rosenfeld LG, Szwarcwald CK. Avaliação da função renal na população adulta brasileira, segundo critérios laboratoriais da Pesquisa Nacional de Saúde. Rev. bras. epidemiol. [Internet]. 2019 [cited 2021 Mar 05]; 22(Supl. 2):E190010.SUPL.2. Available from: http://www.scielo.br/ scielo.php?script=sci_arttext\&pid=S1415-790X2019000300407\&lng=en 